\title{
The Complete Set of Orderable and Deformable Compact Coxeter Hyperbolic Tetrahedrons
}

\author{
Dhrubajit Choudhury \\ The Director, HDR Foundation, Hatigaon, Guwahati, Assam, India
}

\begin{abstract}
We classified the combinatorial structures of 3-dimensional bounded ODCH (orderable and deformable compact hyperbolic) Coxeter polyhedra up to symmetry. Using Plantri graphs and graph theory, we proved that the number of such combinatorial polyhedra is five. One of such combinatorial polyhedra is tetrahedron. Using graph theory and combinatorics, we find that there are six orderable and deformable compact hyperbolic tetrahedra up to symmetry.
\end{abstract}

\section{Introduction}

A $n$-dimensional orbifold is a topological space with a structure based on the quotient space of $R^{n}$ by a finite group action. An orbifold is called good if its universal cover is a manifold. We will concentrate only on good orbifolds.

To give a hyperbolic structure on an orbifold, we model it locally by the orbit spaces of finite subgroups of $P O(1, n)$ acting on open subsets of $\mathrm{H}^{n}$. Similarly, to put a real projective structure on an orbifold, we model it locally by the orbit spaces of finite subgroups of $P G L(n+1, R)$ acting on open subsets of $R P^{n}$.

A real projective structure on an orbifold $M$ implies that $M$ has a universal cover $\tilde{M}$ and the deck transformation group $\pi_{1}(M)$ acting on $\tilde{M}$ so that $\frac{\tilde{M}}{\pi_{1}(M)}$ is homeomorphic to $M$.

A convex set in $R P^{n}$ is a convex set in an affine patch. If we use Klein's model of a $n$-dimensional hyperbolic space, then is an open ball in $R P^{n}$ and $P O(1, n)$ is a subgroup of $P G L(n+1, R)$ preserving $\mathrm{H}^{n}$. Therefore $\mathrm{H}^{n}$ can be imbedded in an $(n+1)$-dimensional real vector space $V$ as an upper part of hyperboloid

$$
-x_{1}^{2}+x_{2}^{2}+\ldots+x_{n+1}^{2}=-1
$$

Hence hyperbolic orbifolds naturally have real projective structures. But a real projective structure of an orbifold may not have hyperbolic structure.

We will concentrate on 3-dimensional compact hyperbolic orbifolds whose base spaces are homeomorphic to a convex polyhedron and whose sides are silvered and each edge is given an order. If the dihedral angle of an edge of a compact hyperbolic polyhedron is $\frac{\pi}{n}$ then we say that the order of the edge is $n$ where $n$ is a positive number.

Definition 1.0.1. Let $X$ be $\mathrm{S}^{3}, \mathrm{E}^{3}$, or $\mathrm{H}^{3}$. Let $\operatorname{Isom}(X)$ denotes the group of isometries of $X$. A Coxeter polyhedron is a convex polytope in $X$ whose dihedral angles are all integer sub-multiples of $\pi$. Let $P$ be a 3dimensional Coxeter polyhedron and $\Gamma$ be the group generated by the reflections in the faces of $P$. Then $\Gamma$ is a discrete group of $\operatorname{Isom}(X)$ and $P$ is its fundamental polyhedron. Conversely, every discrete group $\Gamma$ of $\operatorname{Isom}(X)$ can be obtained from a Coxeter polyhedron $P$ such that $P$ is its fundamental polyhedron. The number of faces intersect at vertex is called the degree of that vertex. Also the edge order of edges of a Coxeter polyhedron are positive integers.

Definition 1.0.2. Let $P$ be a fixed convex polyhedron. Let us assign orders at each edge. Let $e$ be the number of edges and $e_{2}$ be the numbers of order-two. Let $f$ be number of sides.

We remove any vertex of $P$ which has more than three edges ending or with orders of the edges ending there is not of the form $(2,2, n), n \geq 2,(2,3,3),(2,3,4),(2,3,5)$,

i.e., orders of spherical triangular groups. This make $P$ into an open 3-dimensional orbifold. 
Let $\hat{P}$ denote the differential orbifold with sides silvered and the edge orders realized as assigned from $P$ with vertices removed. We say that $\hat{P}$ has a Coxeter orbifold structure.

Definition 1.0.3. The deformation space $\hat{P}$ of projective structures on an orbifold $\hat{P}$ is the space of all projective structures on $\hat{P}$ quotient by isotopy group actions of $\hat{P}$.

Definition 1.0.4. We say $P$ is orderable if we can order the sides of $P$ so that each sides meets sides meets sides of higher index in less than or equal to 3 edges.

Example 1.0.5. Cube and dodecahedron are not satisfying orderability condition.

Definition 1.0.6. Let $\hat{P}$ be the orbifold structure of a 3-dimensional polyhedron $P$. We say that the orbifold structure $\hat{P}$ is orderable if the sides of $P$ can be ordered so that each side has no more than three edges which are either of order 2 or included in a side of higher index. $\hat{P}$ is trivalent if each side $F$ has three or less number of edges of order two or edges belonging to sides of higher class than $F$.

Definition 1.0.7. A combinatorial polyhedron is a 3-ball whose boundary sphere $S^{2}$ is equipped with a cell complex whose 0 -cells, 1-cells and 2-cells will also be called vertices, edges and faces respectively, and which can be realized as a convex polyhedron. Topologically, a compact polyhedron $P$ is a combinatorial polyhedron. A polyhedron is called trivalent if degree of each vertex is 3 .

Remark 1.0.8. For our convenient, we will use notation in short as:

i. A compact hyperbolic Coxeter polyhedron is as $\mathrm{CH}-\mathrm{Coxeter} \mathrm{polyhedron.}$

ii. An orderable and projectively deformable compact hyperbolic Coxeter orbifold is as ODCH-Coxeter orbifold.

Theorem 1.0.9. Let $T$ be a compact hyperbolic Coxeter tetrahedron and $\hat{P}$ be its Coxeter orbifold structure. Suppose that $\hat{P}$ is orderable and projectively deformable. Then the total number of such Tis 6 and T is one of the tetrahedrons in figure 1.

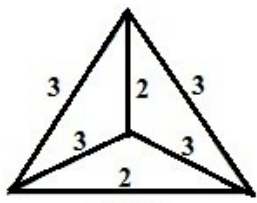

T-1

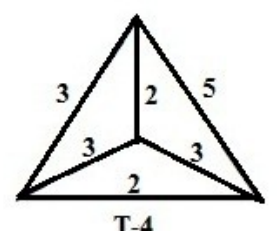

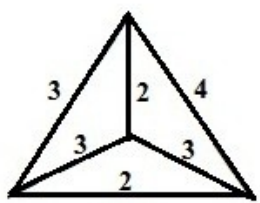

T-2

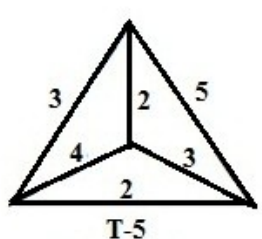

IGURE 1

\section{Figure}

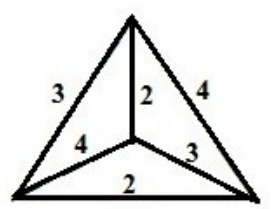

T-3

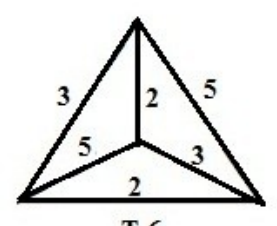

T-6

Proof. Using Choi's theorem, we prove that the number of edges of order 2 is exactly 2. By Andreev's condition for compact hyperbolic polyhedra, we prove that the order of the edges at each vertex is one of the form $(2,3,3),(2,3,4),(2,3,5)$. Using graphical properties, we can assign the orders to each edge in six different ways up to symmetry. Therefore the number of orderable and deformable compact hyperbolic tetrahedra is six and these are in figure 1.

\section{Preliminary}

2.1. Andreev's Theorem. In 1970, E.M. Andreev provides a complete characterization of 3-dimensional compact hyperbolic polyhedral having non-obtuse dihedral angles on his article [2]. Therefore Andreev's theorem is a fundamental tool for classification of 3-dimensionalcompact hyperbolic Coxeter polyhedron. Some elementary faces about polyhedral are essential before we state Andreev's theorem.

Definition 2.1.1. A cell complex on $\mathrm{S}^{2}$ is called trivalent if each vertex is the intersection of three faces. A 3dimensional combinatorial polyhedron is a cell complex $C$ on $\mathrm{S}^{2}$ that satisfied the following condition:

i. Every edges of $C$ is the intersection of exactly two faces.

ii. Anon-empty intersection of two faces is either an edge or a vertex. 
iii. Every face contains not fewer than 3 edges. If a face contains $n$ edges then $n$ is called the length of the face. Suppose $C^{*}$ be the dual complex of $C$ in $\mathrm{S}^{2}$. Then $C^{*}$ is a simplicial complex which embed in the same $\mathrm{S}^{2}$ so that the vertex correspond to face of $C$, etc. A simple closed curve $\Gamma$ in $C^{*}$ is called $k$-circuit if it is formed by $k$ edges of $C^{*}$. A $k$-circuit $\Gamma$ is called prismatic $k$-circuit if the intersection of any two edges of $C$ intersected by $\Gamma$ is empty. If a prismatic $k$-circuit meets the edges $e_{1}, e_{2}, \ldots, e_{k}$ of $C$ successively then we say that the edges $F_{1}, F_{2}, \ldots, F_{k}$ are an $k$-prismatic element of $C$.

Theorem 2.1.2 (Andreev, 1970), Let $C$ be an combinatorial polyhedron such that $C$ is not a simplex and suppose that non-obtuse angles $0<\alpha_{i j} \leq \frac{\pi}{2}$ are given corresponding to each edge $F_{i j}=F_{i} \cap F_{j}$ of $C$ where $F_{i}$ and $F_{j}$ are the faces of $C$. Then there exist a compact hyperbolic polyhedron $P$ in 3-dimensional hyperbolic space which realize $C$ with dihedral angles $\alpha_{i j}$ at the edge $F_{i j}$ if and only if the following five conditions hold:

i. $C$ is trivalent.

ii. If $F_{i j}=F_{i} \cap F_{j} \cap F_{k}$ is a vertexr of $C$ then $\alpha_{i j}+\alpha_{j k}+\alpha_{k i}>\pi$.

iii. If $\Gamma$ is a prismatic 3-circuit which intersects edges $F_{i j}, F_{j k}, F_{k i}$ of $C$ then $\alpha_{i j}+\alpha_{j k}+\alpha_{k i}<\pi$

iv. If $\Gamma$ is a prismatic 4-circuit which intersects edges $F_{i j}, F_{j k}, F_{k i}, F_{l i}$ of $C$ then

$\alpha_{i j}+\alpha_{j k}+\alpha_{k l}+\alpha_{l i}<2 \pi$.

v. If $F_{s}$ is a four sides faces of $C$ with edges $F_{i s}, F_{j s}, F_{k s}, F_{l s}$ enumerated successively, then

$\alpha_{i s}+\alpha_{k s}+\alpha_{i j}+\alpha_{j k}+\alpha_{k l}+\alpha_{l i}<3 \pi, \alpha_{j s}+\alpha_{l s}+\alpha_{i j}+\alpha_{j k}+\alpha_{k l}+\alpha_{l i}<3 \pi$

Furthermore, this polyhedron is unique up to hyperbolic isometries. Also Roeder, Hubbard and Dunbar proved that if $C$ is not a triangular prism, then condition (5) is a consequence of (3) and (4) (Sec [14]). Andreev's restriction to non-obtuse dihedral angles is necessary to ensure that $P$ be convex. Without this restriction of dihedral angles, compact hyperbolic polyhedral realizing a given abstract polyhedron may not be convex. Since dihedral angles of Coxeter polyhedron is non-obtuse, Andreev's theorem provide a complete characterization of 3-dimensional hyperbolic Coxeter polyhedron having more than four faces.

2.2. Choi's Theorem. Prof. Choi concentrated a class of Coxeter orbifolds which is called orderable Coxeter orbifolds and a certain type of orbifolds known as normal type orbifolds. In this class of orbifolds, we understand the restricted deformation space of orbifolds in real projective space from his article [6].

Definitation 2.2.1. We denote $k(P)$ the dimension of the projective group acting on a convex polyhedron $P$.

$$
k(P)=\left\{\begin{array}{l}
3 \text { if } P \text { is a tetrahedron, } \\
1 \text { if } P \text { is a cone with base } \\
\quad \text { a convex polygon which is no } \\
0 \text { otherwise }
\end{array}\right.
$$

Definition 2.2.2. A Coxeter group $\Gamma$ is an abstract group define by a group presentation of form

$$
\left(R_{i,} ;\left(R_{i} R_{j}\right)^{n_{i j}}\right), i, j \in I \text {. }
$$

Where $I$ is a countable index set, $n_{i j} \in N$ is symmetric for $i, j$ and $n_{i j}=1$.

The fundamental group of the orbifold will be a Coxeter group with a presentation

$$
R_{i}, i=1,2, \ldots f,\left(R_{i} R_{j}\right)^{n_{i j}}=1
$$

where $R_{i}$ is associated with silvered sides and $R_{i, j}$ has order $n_{i, j}$ associated with the edge formed by the $i$-th and $j$-th side meeting.

A Coxeter orbifold whose polytope has a side $F$ and a vertex $v$ where all other sides are adjacent triangles to $F$ and contains $v$ and all edge orders of $F$ are 2 is called a cone-type Coxeter orbifold. A Coxeter orbifold whose polyhedron is topologically a polygon times an interval and edges orders of top and bottom sides are 2 is called a product-type Coxeter orbifold. If $\hat{P}$ is not above type and has not finite fundamental group, then $\hat{P}$ is said to be a normal-type Coxeter orbifold. 
Theorem 2.2.3 (Choi, 2006). Let $P$ be a convex polyhedron and $\hat{P}$ be given a normal type Coxeter orbifold structure. Let $k(P)$ be the dimension of the group of projective automorphisms acting on P. Suppose that $\hat{P}$ is orderable. Then the restricted deformation space of projective structures on the orbifold $\hat{P}$ is a smooth manifold of dimension $3 f-e-e_{2}-k(P)$ if it is not empty.

Remark 2.2.4. Let $P$ be a convex polyhedron and $\hat{P}$ be given a normal type Coxeter orbifold structure. Let $k(P)$ be the dimension of the group of projective automorphisms acting on $P$. Suppose that $\hat{P}$ is orderable. Then $\hat{P}$ is projectively deformable if and only if $3 f-e-e_{2}-k(P)>0$.

2.3. Planar Graphs. The study of graphs is very important to understand the combinatorial structure of a polyhedron. We will discuss about the basis relation between graph theory and the 3-dimensional convex polyhedron.

Definition 2.3.1. A planar graph is a graph that can be drawn on the sphere( or the plane) without edge crossings. Two edges of a graph are parallel if they have the same endpoints. A loop is an edge whose endpoints are the same vertex. If there are neither parallel edges nor loops, a graph is called simple. A simple graph is called $k$-connected if the removal of any $k-1$ or fewer vertices (all the edges they are incident with) leaves a connected graph. The dual graph of a plane graph is a plane graph obtained from the original graph by exchanging the vertices and faces. The dual graph of a graph is $k$-connected if and only if the graph is $k$ connected. If all the faces of planar graph is triangles then the graph is called triangulation. The dual of a triangulation is a trivalent planar graph. A triangulation with $n$ vertices has exactly $3 n-6$ edges and $2 n-4$ faces.

Definition 2.3.2. Let $G_{1}=\left(V_{1}, E_{1}\right)$ and $G_{2}=\left(V_{2}, E_{2}\right)$ be two graphs imbedded on the sphere such that $V_{1}, V_{2}$ be the set of vertices of $G_{1}, G_{2}$ and $E_{1}, E_{2}$ be the sets of edges of $G_{1}, G_{2}$. An isomorphism from $G_{1}$ to $G_{2}$ is a pair of bijections $\varphi: V_{1} \rightarrow V_{2}$ and $\varphi: E_{1} \rightarrow E_{2}$ which preserve the vertex-edge incidence relationship.

Definition 2.3.3. Let $P$ be a convex polyhedron. The vertices and the edges of $P$ from an abstract, finite, simple graph, called the graph of $P$ and denoted by $G(P)$. Thus, $G(P)$ is an abstract graph defined on the set of vertices vert $(P)$ of $P$. Two vertices $u$ and $v$ in vert $(P)$ are adjacent in $G(P)$ if and only if $[u, v]$ is an edge of $P$.

Definition 2.3.4. A 3-dimensional polyhedron is called simplicial polyhedron if every face contain exactly 3 vertices. A 3-dimensional polyhedron is called a simple polyhedron if each vertex is the intersection of exactly 3 faces.

Theorem 2.3.5 (Blind and Mani). If $P$ is convex polyhedron, then the graph $G(P)$ determines the entire combinatorial structure of $P$.

In other words, if two simple polyhedral have isomorphic graphs, then their combinatorial polyhedral are isomorphic as well.

Steinitz established the following basic theory for 3-dimensional polyhedron.

Theorem 2.3.6 (Steinitz). $G(P)$ is the graph of a 3-dimensional polyhedron $P$ if and only if it is simple, planar and 3-connected.

Corollary 2.3.7. Every 3-connected planar graph has a representation in the plane such that all edges are straight, and all the bounded regions determined by it, as well as the union of all the bounded regions, are convex polygons.

Since the compact hyperbolic polyhedron is simple, the combinatorial polyhedron of a compact hyperbolic polyhedron can be known from 3-connected planar graph of the polyhedron. 


\section{Results}

3.1. Known Results from previous article. In my previous articles [The graphical investigation of orderable and deformable compact Coxeter polyhedral in hyperbolic space] and [Application of Plantri graph: All Combinatorial structure of Orderable and Deformable Compact Coxeter Hyperbolic Polyhedra], we found the following theorems and propositions:

Let $P$ be a CH-Coxeter polyhedron and $\hat{P}$ be its Coxeter orbifold structure of $P$.

Proposition 3.1.1. If $\hat{P}$ is orderable, then $P$ is also orderable.

Remark 3.1.2. If $P$ is not orderable, then $\hat{P}$ is also not orderable.

Let $e$ be the number of edges of $P$ and $e_{2}$ be the number of edges of edge order 2. Let $v$ be the number of vertices of $P$ and $f$ be the number of faces of $P$.

Proposition 3.1.3. Let $P$ be a 3-dimensional compact hyperbolic Coxeter polyhedron and $\hat{P}$ be Coxeter orbifold structure of $P$. Suppose $\hat{P}$ is orderable and projectively deformable. Then

a. Every vertex is incident with exactly three edges.

b. Every vertex is incident with at least one edge of edge order 2.

c. $\frac{v}{2} \leq e_{2} \leq 5 \Rightarrow v \leq 10$.

d. $v$ is even.

Proposition 3.1.4. Let $P$ be a 3-dimensional CH-Coxeter polyhedron and $\hat{P}$ be its Coxeter orbifold structure. Suppose that $\hat{P}$ is orderable and projectively deformable. Then the total number of combinatorial polyhedral of such $P$ is 5 and $P$ is one of the combinatorial polyhedral in figure 2.

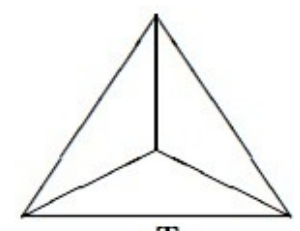

$\mathrm{T}$

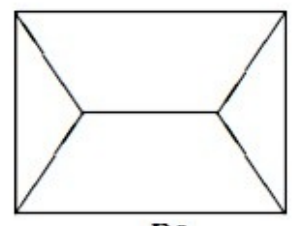

P6

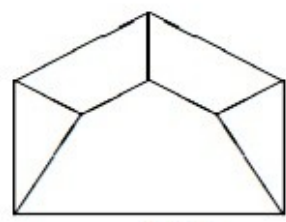

P8-1

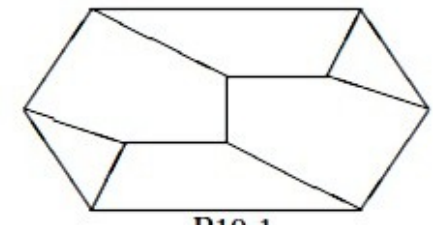

P10-1

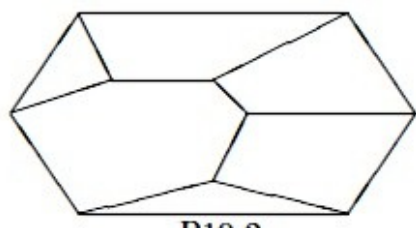

P10-2

Figure 2

3.2 Main Results. Now we are ready to establish the main results.

Proposition 3.2.1. Let $T$ be a 3-dimensional CH-Coxeter tetrahedron and $\hat{P}$ be its Coxeter orbifold structure of $P$. If $\hat{P}$ is orderable and projectively deformable then $e_{2}=2$.

Proof. By Choi's theorem 2.2.3, $3 f-e-e_{2}-k(T)>0$. By definition 2.2.1, for the tetrahedron $T, k(T)=3$. Since $f=4, e=6$, therefore $3 f-e-e_{2}-k(T)>0 \Rightarrow 3 \cdot 4-6-e_{2}-3>0 \Rightarrow 3>e_{2}$

By proposition 3.1.3, every vertex is incident with at least one edge of edge order 2. Since there are 4 vertices of $T$ and each vertex incident with exactly two vertices, therefore the number of edges of order 2 is at least $\frac{v}{2}=2$.

Hence $e_{2} \geq 2$. Since $2 \leq e_{2}<3$, therefore $e_{2}=2$. 
Proposition 3.2.2. Let $\mathrm{T}$ be a 3-dimensional $\mathrm{CH}$-Coxeter tetrahedron and $\hat{P}$ be its Coxeter orbifold structure of $P$. If $\hat{P}$ is orderable and projectively deformable then the order of the edges at each vertex is one of the form $(2,3,3),(2,3,4),(2,3,5)$.

Proof. Since there are two edges of $T$ of order 2 and 4 vertices in $T$ and each vertex is incident with at least one edge of order 2 , therefore each vertex is incident with exactly one edge of order 2.

Suppose that $r_{1}, r_{2}$ be the order of the two edges at a vertex other than 2 . Then $r_{1}, r_{2} \geq 3$.

By Andreev's first condition, we have

$$
\frac{1}{2}+\frac{1}{r_{1}}+\frac{1}{r_{2}}>1 \Rightarrow \frac{1}{r_{1}}+\frac{1}{r_{2}}>\frac{1}{2}
$$

Since $r_{1}, r_{2} \geq 3$, therefore $\frac{1}{r_{1}}+\frac{1}{r_{2}}<\frac{2}{3}$. Hence $\frac{1}{2}<\frac{1}{r_{1}}+\frac{1}{r_{2}} \leq \frac{2}{3}$.

If $r_{1}, r_{2} \geq 4$ then $\frac{1}{r_{1}}+\frac{1}{r_{2}} \leq \frac{1}{2}$. This is a contradiction. Hence $r_{1}=3$ or $r_{2}=3$.

Assume that $r_{2}=3$. If $r_{1} \geq 6$ then

$$
\frac{1}{2}<\frac{1}{r_{1}}+\frac{1}{r_{2}} \Rightarrow \frac{1}{2}<\frac{1}{6}+\frac{1}{r_{2}} \Rightarrow \frac{1}{2}-\frac{1}{6}<\frac{1}{r_{2}} \Rightarrow \frac{2}{6}<\frac{1}{r_{2}} \Rightarrow r_{2}<3 \Rightarrow r_{2}=2
$$

This is a contradiction. Hence $3 \leq r_{1} \leq 5$.

Therefore the edge order at each vertex is one of the form $(2,3,3),(2,3,4),(2,3,5)$.

Theorem 3.2.3. Let $T$ be a compact hyperbolic Coxeter tetrahedron and $\hat{P}$ be its Coxeter orbifold structure. Suppose that $\hat{P}$ is orderable and projectively deformable. Then the total number of such $T$ is 6 and $T$ is one of the tetrahedrons in figure 1.

Proof. Since there are two edges of order 2 and each vertex is incident with an edge of order 2, therefore both the edges of order 2 are disjoint. Up to symmetry, we can assign the edge order 2 as follows:

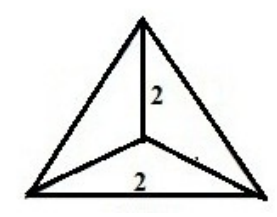

Since edge orders at each vertex is one of the form $(2,3,3),(2,3,4),(2,3,5)$, therefore we assign 3 as the order of edge up to symmetry for each vertex as follows:

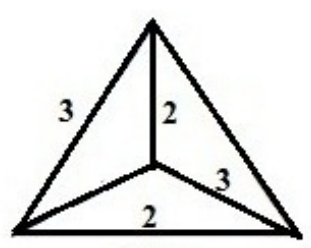

Up to symmetry, we can assign the order to the remaining two edges as $(3,3),(3,4),(3,5),(4,4),(4,5),(5,5)$. Therefore the tetrahedrons with edges order are as in figure 1. 


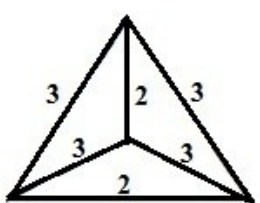

T-1

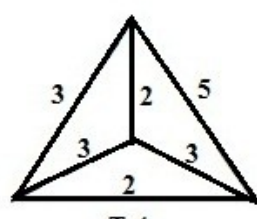

T-4

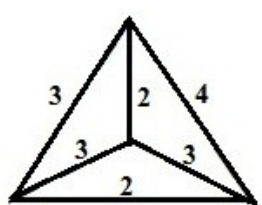

T-2

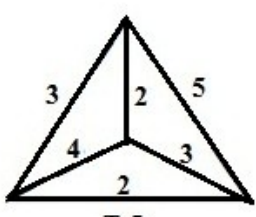

T-5

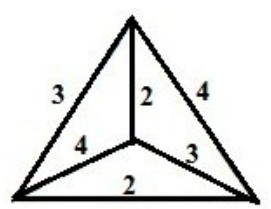

T-3

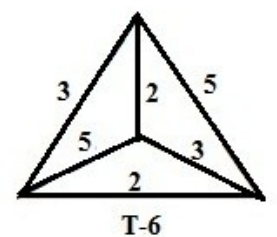

T-6

Figure 1

Since each face is triangular, therefore we can assign order of the faces randomly.

\section{Conclusion}

In this article, we proved that the number of orderable and deformable compact hyperbolic Coxeter tetrahedrons in real projective space is exactly six. It can be extended to find all the 3-dimensional compact hyperbolic Coxeter polyhedral which are orderable and deformable in real projective space.

\section{References}

[1] Dhrubajit Choudhury. The graphical investigation of orderable and deformable compact Coxeter polyhedral in hyperbolic space.

[2] Dhrubajit Choudhury, Pranab Kalita. Application of Plantri graph: All Combinatorial structure of Orderable and Deformable Compact Coxeter Hyperbolic Polyhedra. IOSR Journal of Mathematics (IOSR-JM), e-ISSN: 2278-5728, p-ISSN: 2319-765X, Volume 7, Issue 3, PP 63-67.

[3] E.M. Andreev. On convex polyhedral of finite volume in Lobacevskii space. Math. USSR Sbornik 10, 413-440 (1970)

[4] E.M. Andreev. On convex polyhedral of finite volume in Lobacevskii space. Math. USSR Sbornik 12, 255-259 (1970)

[5] S. Choi. Geometric Structures on Orbifolds and Holonomy Representations. Geom. Dedicata 104, 161-199 (2004)

[6] S. Choi, W.M. Goldman. The deformation spaces of convex $R P^{2}$-structures on 2-orbifolds. Amer. J. Math. 127, 1019-1102 (2005)

[7] S. Choi. The deformation spaces of projective structures on 3-dimensional Coxeter orbifolds. Geom. Dedicata 119, 69-90 (2006)

[8] D. Cooper, D. Long, M. Thistlethwaite. Computing varieties of representation s of hyperbolic 3 -manifolds into $\operatorname{SL}(4, R)$. Experiment. Math. 15,291-305 (2006)

[9] H. Garland. A rigidity theorem for discrete subgroups. Trans. Amer. Math. Soc. 129, 1-25 (1967)

[10] W.M. Goldman. Convex real projective structures on compact surfaces. J. Differential Geom. 31,791-845 (1990)

[11] V.G. Kac, E.B Vinberg. Quasi-homogeneous cones. Math. Zamnetki 1, 347-354 (1967)

[12] R.K.W. Roeder. Constructing hyperbolic polyhedral using Newton's method. Experiment. Math. 16, $463-492$ (2007)

[13] Roland K.W. Roeder, John H. Hubbard and William D. Dunbar. Andreev's Theorem on hyperbolic polyhydra. Ann. Inst. Fourier (Grenoble) 57(2007), no.3,825-882.

[14] Anna Felikson, Pavel Tumaarkin. Coxeter polytopes with a unique pair of non intersecting facets. Journal of Combinatorial Theory, Series A 116(2009) 875-902.

[15] E. B. Vinberg. Discrete linear groups that are generated by reflections. Izv. Akad. Nauk SSSR 35, 1072-112 (1971)

[16] E.B. Vinberg. Hyperbolic reflection groups. Uspekhi Mat. Nauk 40, 29-66 (1985)

[17] E.B. Vinberg. Geometry II. Encyclopedia of Maths. Sc. 29. Springer 1993.

[18] A. Weil. On Discrete subgroups of Lie groups. Ann. of Math. 72, 369-384 (1960)

[19] A. Weil. On Discrete subgroups of Lie groups II. Ann. of Math. 75,578-602 (1962) 